2009-01-01

\title{
Misalignment Limits for a Singlemode-multimode-singlemode Fiber-based Edge Filter
}

\author{
Agus Hatta \\ Technological University Dublin, ahatta@tudublin.ie \\ Gerald Farrell \\ Technological University Dublin, gerald.farrell@tudublin.ie \\ Pengfei Wang \\ Technological University Dublin, pwang@tudublin.ie
}

See next page for additional authors

Follow this and additional works at: https://arrow.tudublin.ie/engscheceart

Part of the Electrical and Computer Engineering Commons

\section{Recommended Citation}

Hatta, A. et al (2009) Misalignment limits for a singlemode-multimode-singlemode fiber-based edge filter. Journal of Lightwave Technology, Vol.27, no.13, pp.2482-2488. DOI:10.1109/JLT.2008.2011499

This Article is brought to you for free and open access by the School of Electrical and Electronic Engineering at ARROW@TU Dublin. It has been accepted for inclusion in Articles by an authorized administrator of ARROW@TU Dublin. For more information, please contact arrow.admin@tudublin.ie, aisling.coyne@tudublin.ie, gerard.connolly@tudublin.ie.

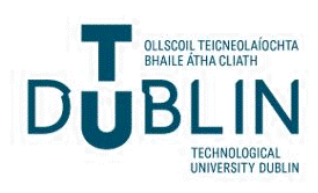




\section{Authors}

Agus Hatta, Gerald Farrell, Pengfei Wang, Ginu Rajan, and Yuliya Semenova

This article is available at ARROW@TU Dublin: https://arrow.tudublin.ie/engscheceart/43 


\title{
Misalignment Limits for a Singlemode-Multi- mode-Singlemode Fiber-Based Edge Filter
}

\author{
Agus Muhamad Hatta, Gerald Farrell, Pengfei Wang, Ginu Rajan, and Yuliya Semenova
}

\begin{abstract}
Misalignment effects on the spectral characteristics of edge filters based on singlemode-multimode-singlemode (SMS) fibre structures are investigated numerically and experimentally. A modal propagation analysis is used with a set of guided modes calculated using the finite-difference method to determine the transmission loss of the SMS-based edge filters. A limit for the tolerable misalignment of the SMS fiber-based edge filter is proposed, beyond which the spectral performance of the SMS structure degrades unacceptably. The numerical results are verified experimentally with good agreement.
\end{abstract}

Index Terms-Edge filter, multimode interference, optical fibers.

\section{INTRODUCTION}

$\mathbf{S}$ INGLEMODE-MULTIMODE-SINGLEMODE (SMS) fiber structures have been investigated for use in several applications, e.g., as a refractometer, a bandpass filter, and an edge filter [1]-[4]. An optical device based on the SMS fiber structure offers an all-fiber solution for optical communications and optical sensing applications with the advantages of simplicity of packaging and ease of interconnection to other optical fibers.

The SMS structure is fabricated by splicing a precisely dimensioned multimode fiber (MMF) section between two singlemode fibers (SMFs). Ideally, the center axes of all the fibre cores are precisely aligned. However, in practice the splicing process itself, along with the manufacturing variations in core-cladding concentricity can introduce lateral misalignment between the centers of the SMF-MMF-SMF cores.

In [2], [4], and [5], the SMS fiber structure is analyzed using a modal propagation analysis (MPA) for the linearly polarized (LP) (or scalar) modes. The input light can be assumed to have the field distribution of the fundamental mode of the SMF [5]. When the light launches into the MMF, the input field can be decomposed into the eigenmodes $\left(\mathrm{LP}_{n m}\right)$ of the MMF. Due to the circular symmetric nature of the input field and an ideal alignment assumption, the number of guided modes of the MMF

Manuscript received September 12, 2008; revised December 04, 2008. First published April 17, 2009; current version published July 01, 2009.

A.M. Hatta is with the Applied Optoelectronics Centre, School of Electronic and Communications Engineering, Dublin Institute of Technology, Kevin Street, D8, Ireland, on leave from Engineering Physics Department, Institut Teknologi Sepuluh Nopember (ITS), Surabaya, Indonesia (e-mail: ahatta@dit.ie).

G. Farrell, P. Wang, G. Rajan, and Y. Semenova are with the Applied Optoelectronics Centre, School of Electronic and Communications Engineering, Dublin Institute of Technology, Dublin D8, Ireland (e-mail: gerald.farrell@dit.ie; pwang@dit.ie; ginu.rajan@dit.ie; yuliya.semenova@dit.ie).

Color versions of one or more of the figures in this paper are available online at http://ieeexplore.iee.org.

Digital Object Identifier 10.1109/JLT.2008.2011499 used in the modal propagation analysis is greatly reduced from $\mathrm{LP}_{n m}$ to $\mathrm{LP}_{0 m}$ or the circular symmetry modes. This reduced number of modes means the calculation can be performed efficiently. In [1] and [3], the SMS structure is investigated using the beam propagation method, where it is assumed that only the circular symmetry modes exist. With this assumption the optical field is simplified so that it is independent of the angular coordinate in a cylindrical coordinate system. However, if the center (or meridional) axes of the SMS cores are misaligned relative to one another then we cannot assume circularly symmetrical modes. Thus, both approaches published so far cannot be used to study the effect of misalignment in an SMS structure.

An MPA using a complete set of hybrid modes or vectorial form guided modes in the MMF has been proposed to analyze the misalignment effect [6]. In this approach, a complete set of guided modes in the MMF is calculated and an adaptive algorithm is developed to perform mode expansion of the optical field in the MMF. However, the complete set of guided modes in the MMF can also be solved with an alternative numerical method, the finite difference method (FDM) [7]. The numerical approach using FDM offers simplicity of its implementation. In this paper, the FDM is used to calculate the complete set of guided modes in the MMF and then the MPA is performed to analyze the misalignment effect. Building on previous research on an SMS-based edge filter [3], [4], in this paper, the effect of fiber misalignment within an SMS-based edge filter is investigated both numerically and experimentally, so as to establish an upper limit on tolerable misalignment above which the performance of SMS structure is degraded significantly.

To put the misalignment induced performance degradation in context, the application chosen here for the SMS is that of an edge filter used within a ratiometric wavelength measurement system. A ratiometric wavelength measurement usually consists of a 3-dB coupler which the two outputs connect to an edge filter arm with a well-defined spectral response and a reference arm, or alternatively two edge filter arms with opposite slope spectral responses can be used. The use of two opposite slope edge filters can increase the usable resolution of the ratiometric system [8]. A ratiometric wavelength measurement based system on two opposite slope SMS-based edge filters is built and demonstrated in this paper.

\section{SMS-BASED EDGE FILTERS}

A schematic structure for a ratiometric wavelength measurement consisting of two SMS-based edge filters is shown in Fig. 1(a). The target spectral responses in $\mathrm{dB}$ of the SMS-based edge filters are shown in Fig. 1(b), and can have either a negative (P1) or a positive (P2) slope. Two key parameters for an edge filter are baseline loss and discrimination range. The 


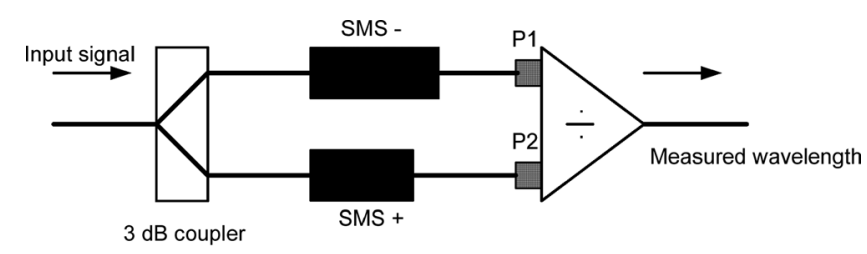

(a)

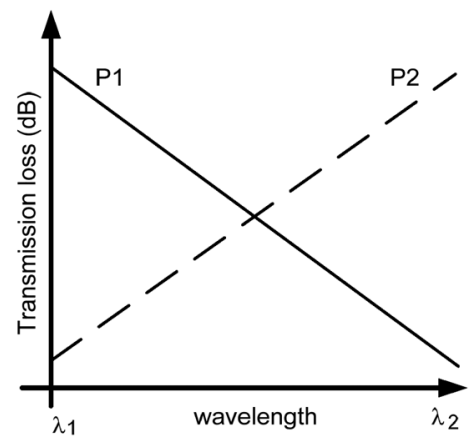

(b)

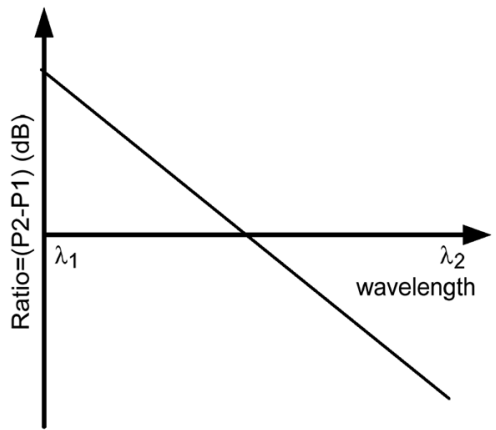

(c)

Fig. 1. (a) Schematic configuration of a ratiometric wavelength measurement, (b) desired spectral responses of the SMS-based edge filter, negative (solid line) and positive (dash line) slope versions, and (c) the output ratio between two output SMS-based edge filters.

SMS-based edge filter operates over a wavelength range from $\lambda_{1}$ to $\lambda_{2}$ with a progressively larger or smaller transmission loss as the wavelength increases from $\lambda_{1}$ to $\lambda_{2}$, for the negative or positive slope, respectively. The baseline loss is defined as the transmission loss of the filter at $\lambda_{1}$ or $\lambda_{2}$, for the negative and the positive slopes, respectively, while the discrimination range is the difference between the transmission loss at $\lambda_{1}$ and $\lambda_{2}$. The corresponding ratio (P2-P1) of the two outputs over the wavelength range is presented in Fig. 1(c). The wavelength of an input signal can be determined through measuring the power ratio of the output ports at the outputs of the two arms, assuming that a suitable calibration has taken place.

The fiber structure under consideration consists of an input SMF, a sandwiched MMF section, and an output SMF, as shown in Fig. 2(a). The concentric alignment and misalignment conditions in the Cartesian coordinate system, between the input SMF, MMF section, and output SMF cores, are shown in Fig. 2(b) and (c), respectively. The radii of SMF and MMF are denoted as $R_{s}$ and $R_{m}$, respectively. The input SMF and

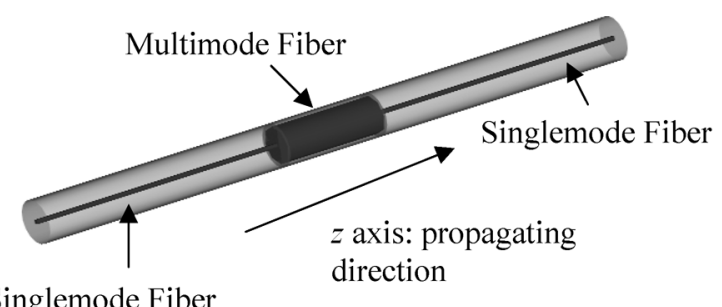

Singlemode Fiber

(a)

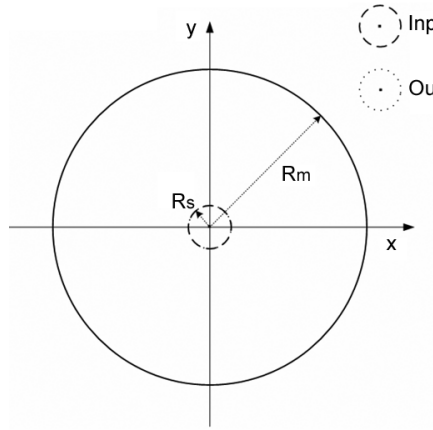

(b)

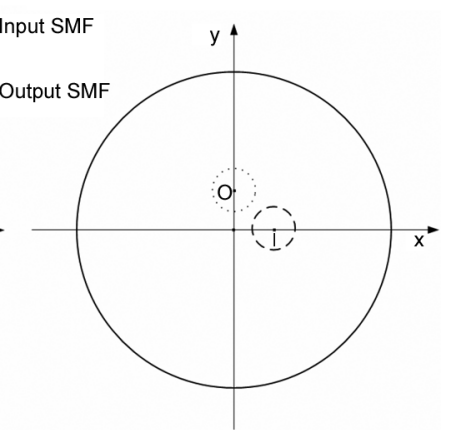

(c)
Fig. 2. (a) Schematic configuration of the SMS fiber structure, (b) concentric alignment, and (c) misalignment condition.

output SMF positions are denoted by the coordinates $I[x, y]$ and $O[x, y]$, respectively, where $x$ and $y$ are in $\mu \mathrm{m}$.

\section{Modal Propagation ANALYsis}

The MMF section can support many guided modes and the input field is reproduced as single image at periodic intervals along the propagation direction due to the interference between these guided modes. This is the so-called self-imaging principle and the distance at which self-imaging occurs is called the re-imaging distance. The approach used here to analyze the field distribution in the MMF section is a modal propagation analysis [9]. In the MMF, an MPA using a cylindrical coordinate system has been employed in [2], [4], and [5] based on a scalar approximation of the $\mathrm{LP}_{0 m}$ modes. The $\mathrm{LP}_{0 m}$ modes could not be used to investigate misalignment effects because it only consists of circularly symmetrical modes. To analyze misalignment it is necessary to calculate a complete set of guided modes in the MMF [6]. In the approach used here, the MPA is performed in the Cartesian coordinate system with a set of calculated guided modes using FDM to allow investigation of misalignment effects.

The MPA procedure is as follows: the input light is assumed to have the field distribution $\psi(x, y, 0)$ of the fundamental mode of the SMF. The input field can be decomposed into the eigenmodes of the MMF, $\varphi_{\nu}(x, y)$, when the light enters the MMF section. The input field at the MMF can be written as

$$
\psi(x, y, 0)=\sum_{\nu} c_{\nu} \varphi_{\nu}(x, y)
$$


where $c_{\nu}$ is the excitation coefficient of each mode. The coefficient $c_{\nu}$ can be calculated by an overlap integral between $\psi(x, y, 0)$ and $\varphi_{\nu}(x, y)$

$$
c_{v}=\frac{\int \psi(x, y, 0) \varphi_{\nu}(x, y) d x d y}{\sqrt{\int \varphi_{\nu}^{2}(x, y) d x d y}} .
$$

As the light propagates in the MMF section, the field at a propagation distance $z$ can be calculated by

$$
\psi(x, y, z)=\sum_{\nu} c_{\nu} \varphi_{\nu}(x, y) \exp \left[j \beta_{\nu} z\right]
$$

where $\beta_{\nu}$ is the propagation constant of each eigenmode of the MMF. The transmission loss in $\mathrm{dB}$ can be determined by using the overlap integral method between $\psi(x, y, z)$ and the eigenmode of the output SMF $\psi_{o}(x, y)$

$L_{s}(z)=10 \cdot \log _{10}\left(\frac{\left|\int \psi(x, y, z) \psi_{o}(x, y) d x d y\right|^{2}}{\int|\psi(x, y, z)|^{2} d x d y \int\left|\psi_{o}(x, y)\right|^{2} d x d y}\right)$.

Here $\varphi_{\nu}(x, y)$ and $\beta_{\nu}$ can be obtained by using a semivectorial FDM. It should be noted that FDM calculates a set of all possible guided modes in the MMF section, not just concentric circular modes, allowing the transmission loss due to misalignment to be calculated. Using the above equations, the light propagation in the whole structure can be analyzed.

\section{Design Example AND SPECTRAL Response}

To investigate the effect of misalignment, in the first instance it is necessary to present a typical SMS structure designed to meet a target spectral response and calculate its ideal, perfectly aligned, spectral response.

To design the SMS-based edge filter, the MMF length needs to be determined. It has been shown that the re-imaging distance is wavelength dependent [2], [5]. If re-coupling into the output SMF takes place at the re-imaging distance, then the MMF section of the SMS structure has by definition a length equal to the re-coupling distance and operates as a bandpass filter as in [2] and [5]. However, for the purpose of designing an edge filter, the bandpass response can be considered as two spectral responses, on either side of a center wavelength. Consequently, the device can behave as an edge filter for a selected wavelength range. Two SMS-based edge filters with opposite slope spectral responses within a given wavelength range can be obtained by choosing two bandpass filters with appropriate center wavelengths [4].

As an example to illustrate the design process, a target wavelength range for wavelength measurement from $\lambda_{1}=1520 \mathrm{~nm}$ to $\lambda_{2}=1545 \mathrm{~nm}$ is chosen. This range is chosen as it corresponds to the typical center wavelengths for many fiber Bragg grating (FBG) sensors. Based on the target spectral responses as in Fig. 1(b), the SMS-based edge filters are designed with the baseline loss $>-8 \mathrm{~dB}$ and the desired discrimination range $>8 \mathrm{~dB}$. A standard SMF28 fiber is chosen as the SMF, for

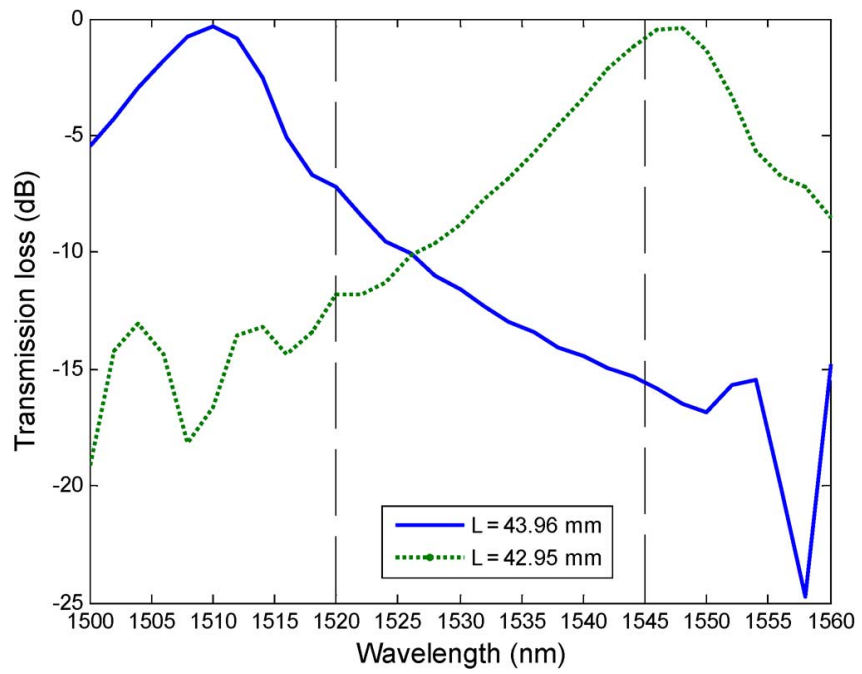

Fig. 3. Transmission loss responses of the two SMS-based edge filters.

which the parameters are: the refractive indices for the core and cladding are 1.4504 and 1.4447, respectively (at a wavelength of $1550 \mathrm{~nm}$ ), and the radius of the core $R_{s}=4.15 \mu \mathrm{m}$. An MMF-type AFS105/125Y is chosen as the MMF section for which the parameters are: refractive indices for the core and cladding are 1.4446 and 1.4271 , respectively, with a core radius $R_{m}=52.5 \mu \mathrm{m}$. The small difference between the refractive indices of the SMF and MMF means that the Fresnel reflection occurring at their interface is negligible (the level of reflection is $-54 \mathrm{~dB}$ or lower relative to the injected light level) and a one-way modal propagation analysis can be used [5]. As mentioned above, for the specified wavelength range, two opposite response slope edge filters (negative and positive slopes) can be obtained by designing two bandpass filters with peak wavelengths: $\leq 1520 \mathrm{~nm}$ and $\geq 1545 \mathrm{~nm}$, respectively. From (10) in [5], the peak wavelengths from 1500 to $1520 \mathrm{~nm}$ correspond to the MMF lengths $L=44.25-43.66 \mathrm{~mm}$, and from 1545 to $1560 \mathrm{~nm}$ correspond to $L=43.00-42.58 \mathrm{~mm}$. Suitable peak wavelengths for the targeted wavelength range are 1510 and $1547 \mathrm{~nm}$ and the corresponding MMF lengths are $L=43.96 \mathrm{~mm}$ and $L=42.95 \mathrm{~mm}$, for the negative and positive slope edge filters, respectively. The peak wavelengths at 1510 and $1547 \mathrm{~nm}$ are chosen for the SMS-based edge filters because their transmission loss responses have a suitable spectral response over the targeted wavelength range from 1520 to $1545 \mathrm{~nm}$. The calculated transmission loss responses by using (4) for the designed SMS-based edge filter are shown in Fig. 3. These responses represent the performance of the design example for the case of perfect alignment. It can be seen that the two opposite edge filter responses within the targeted wavelength range can be achieved using two bandpass filters. The calculated negative and positive slope responses of the SMS-based edge filters from 1520 to $1545 \mathrm{~nm}$ have a transmission loss from -7.20 to $-15.53 \mathrm{~dB}$ and -11.84 to $-0.77 \mathrm{~dB}$, respectively, and the corresponding discrimination ranges are $8.33 \mathrm{~dB}$ and $11.06 \mathrm{~dB}$, respectively, suitable for use as edge filters. 


\section{INVESTIGATION OF MiSALIGNMENT EFFECTS FOR THE DESIGN EXAMPLE}

To investigate the misalignment effect, the transmission loss of several positions of the input SMF and output SMF are calculated using (4). We calculated the transmission loss within the wavelength range $1520-1545 \mathrm{~nm}$ of the offset positions, $I[a, 0]$, $O[0, a]$, where $a=0-10 \mu \mathrm{m}$ with an increment of $2 \mu \mathrm{m}$. Given the need to undertake experimental verification, these misalignment values are chosen based on the deliberate offset that can be produced by the fusion splicer used in the experiments described later. The calculated transmission loss responses are shown in Fig. 4(a) and (b), for the negative and positive slope edge filters, respectively. From Fig. 4(a), for the negative slope, is can be seen that, even with misalignment, the response retains a monotonically decreasing characteristic over the wavelength range and thus is still suitable for use as the edge filter response. However, the discrimination range decreases as the offset increases, from $8.33 \mathrm{~dB}$ without an offset to $8.03,7.41,6.64,5.93$, and $5.15 \mathrm{~dB}$ for an offset equal to $2,4,6,8$, and $10 \mu \mathrm{m}$, respectively. A reduced discrimination range will have a negative impact on measurement accuracy where the edge filter is used within a ratiometric wavelength measurement system. For the positive slope filter, as shown in Fig. 4(b), the response slope changes very significantly when the offset increases. For an offset $a=2$ and $4 \mu \mathrm{m}$ the spectral responses are still suitable as the edge filter, but for larger offset values the transmission loss responses do not monotonically increase across the wavelength range and therefore are not suitable for use as an edge filter.

The change in the negative and positive slopes due to an offset needs to be considered in the context of changes to the overall bandpass responses. The consequences of an increase in the offset on the bandpass response are a shift of the self-imaging position and a reduction in the maximum transmission loss at the peak wavelength of the bandpass filter. Such changes in the overall bandpass response will also clearly change the positive and negative slope responses.

In practice, there is a significant difference between the negative and positive slope responses in terms of the change in the response that is induced by an offset. This difference can be explained as follows. From the MPA discussed above and as described in [6], the presence of an offset for the input SMF increases the number of excitation modes (circularly symmetrical modes and azimuthal modes) compared to the case without an offset (circularly symmetrical modes only). Increasing the number of excitation modes changes the MMF field pattern resulting from interference in the MMF. In turn, the transmission loss, which is a function of the overlap between the MMF field and the eigenmode of the output SMF, varies with changes in the offset of the output SMF.

To better understand the difference in the manner on which an offset affects the negative and positive slopes, the MMF field amplitude profiles for the cases of $a=0 \mu \mathrm{m}$ and $a=10 \mu \mathrm{m}$ are shown in Fig. 5 for a wavelength of $1537 \mathrm{~nm}$. This wavelength is chosen as it corresponds to the wavelength at which the changes in the positive slope are most pronounced, as shown in Fig. 4. The MMF field amplitude profiles for the negative slope response for the case of $a=0 \mu \mathrm{m}$ and $a=10 \mu \mathrm{m}$ are shown in

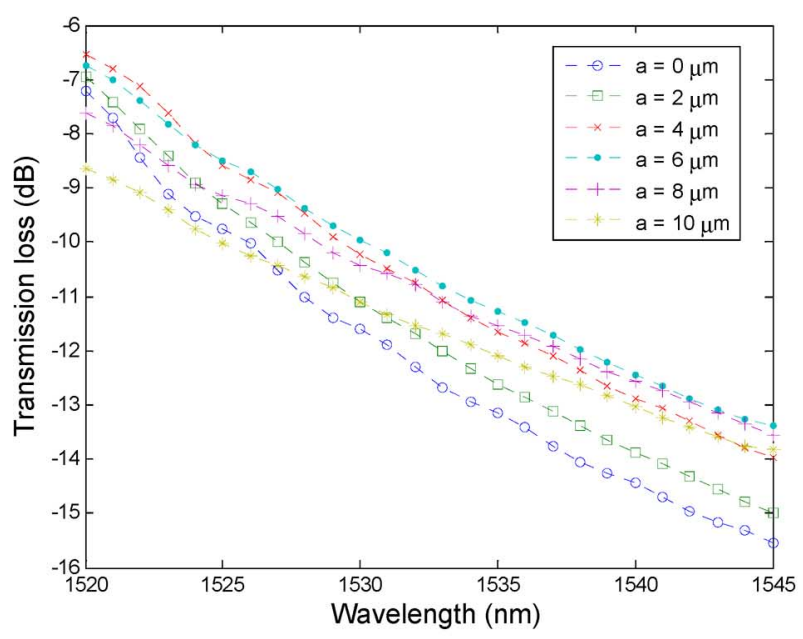

(a)

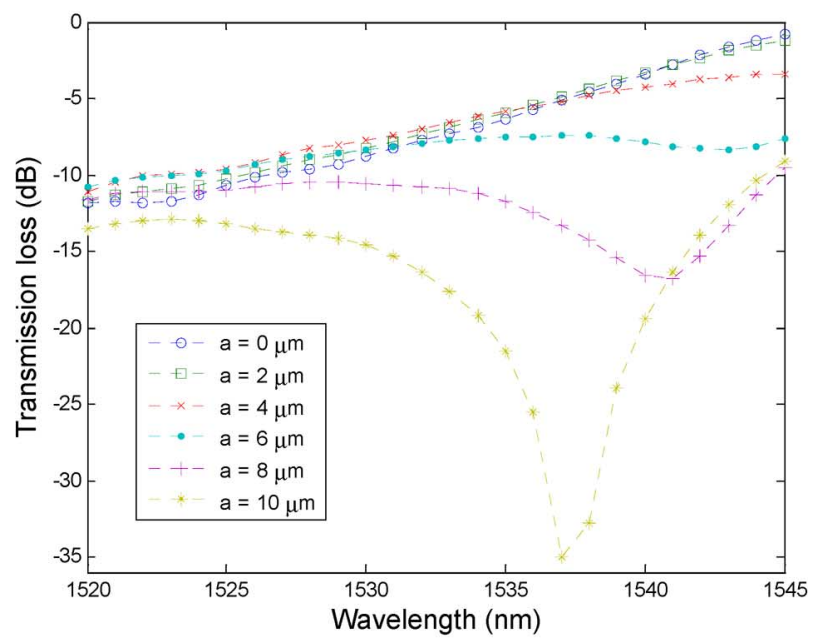

(b)

Fig. 4. Calculated transmission loss response due to misalignment effect of the SMS-based edge filter. (a) Negative slope and (b) positive slope.

Fig. 5(a) and (b), respectively. The overlap between the MMF field amplitude profile and the eigenmode of the output SMF is located at $O[0,0]$ and $O[0,10]$ in $\mu \mathrm{m}$, for the case of $a=0 \mu \mathrm{m}$ and $a=10 \mu \mathrm{m}$, respectively. Comparing Fig. 5(a) and (b), there is only a relatively small difference in the amplitude of the MMF field in the MMF overlap region when an offset is introduced. As a result, the transmission losses are not strongly influenced by offset.

For the positive slope response, the MMF field amplitude profile for the case of $a=0 \mu \mathrm{m}$ and $a=10 \mu \mathrm{m}$ is shown in Fig. 5(c) and (d), respectively. Comparing these figures, it can be seen that when an offset is introduced, that is $a=10 \mu \mathrm{m}$, the eigenmode of the output SMF located at $O[0,10]$ overlaps a portion of the MMF field amplitude profile which has a very low value. The result is a very high transmission loss when an offset is introduced and thus there is a strong dependence of the transmission loss on offset.

To further analyze the spectral quality of the edge filter, it is necessary to examine the linearity of the transmission loss when misalignment occurs. Linearity is important for an edge filter used in wavelength measurement application for two reasons. 


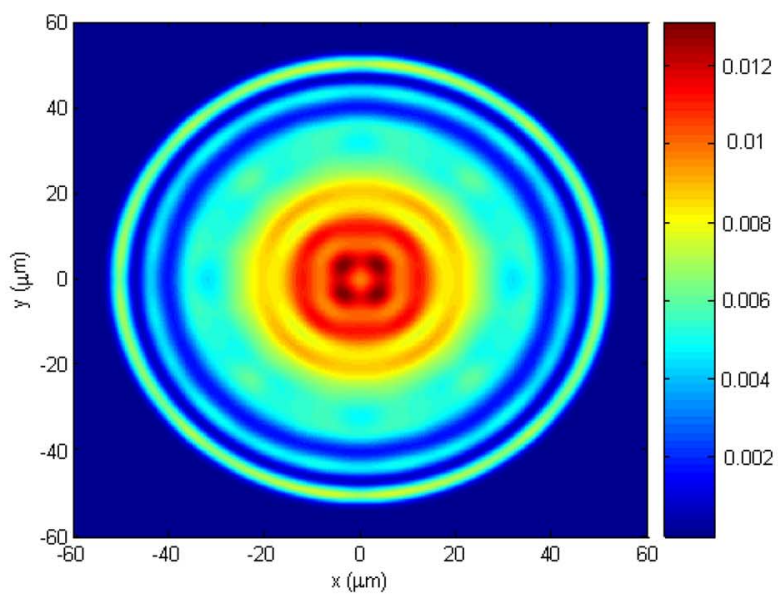

(a)

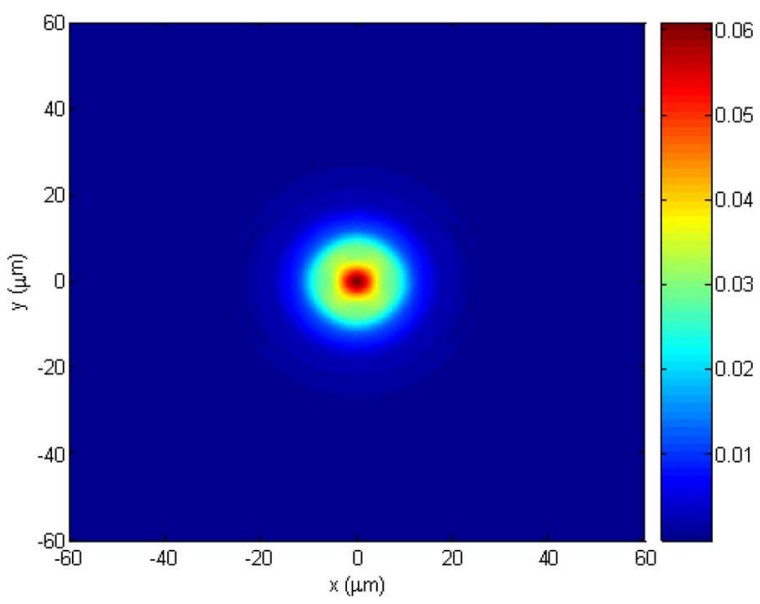

(c)

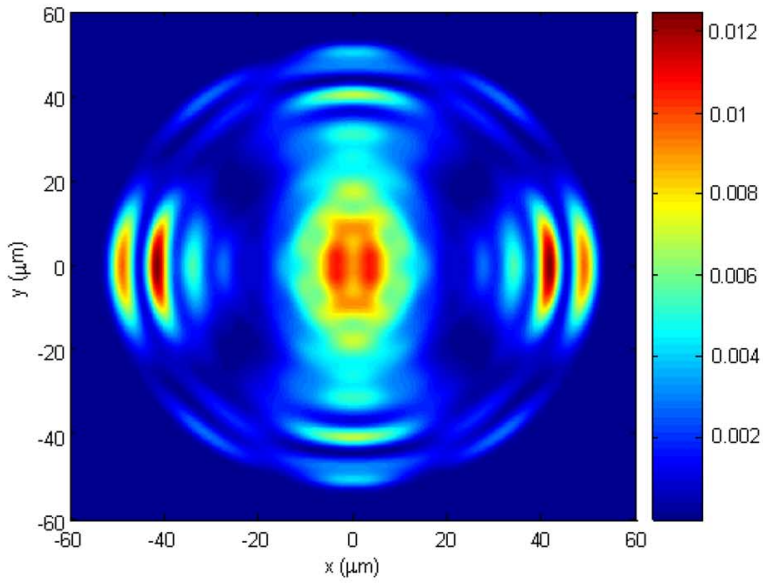

(b)

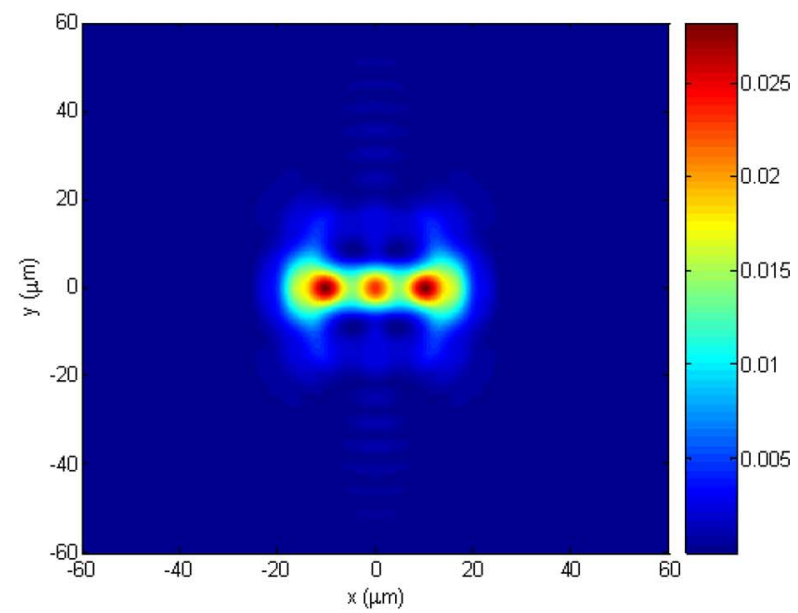

(d)

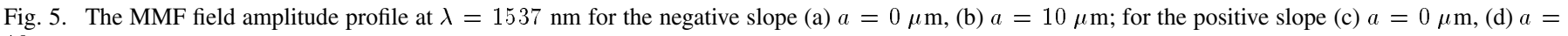
$10 \mu \mathrm{m}$.

First, a linear response by definition monotonically increases or decreases, so there can be no ambiguity in wavelength measurements. Second, a linear response will ensure that the resolution for wavelength measurement is the same for all measured wavelengths. The linearity can be examined by using the correlation coefficient $\left(R^{2}\right)$ of the linear regression analysis. An ideal spectral response has $R^{2}=1$, and a lower $R^{2}<1$ indicates a lower quality for the spectral response linearity. Fig. 6 shows the correlation coefficient for the different offsets from 0 to $10 \mu \mathrm{m}$ with an increment of $1 \mu \mathrm{m}$. It is shown that for the negative slope, the $R^{2}$ maintains a high value and is almost constant with $R^{2}=0.98,0.98,0.99,0.99,0.99,0.99$, and 0.99 for the offset $a=0,2,4,6,8$, and $10 \mu \mathrm{m}$, respectively. This means that the offset has little effect on the slope quality for the negative slope spectral response, but does reduce the discrimination range as mentioned above. For the positive slope, it is clear that the offset effects the slope quality. The $R^{2}$ are $0.98,0.99,0.99$, $0.67,0.28$, and 0.07 for the offset of $0,2,4,6,8$, and $10 \mu \mathrm{m}$, respectively. The $R^{2}$ value degrades beyond an offset value of $a=5 \mu \mathrm{m}$, with $R^{2}=0.95$, and such spectral responses are not suitable for use in an edge filter. Based on the calculation of the $R^{2}$ value, it is suggested as a conservative guiding principle that the misalignment should be less than the core radius of the
SMF (4.15 $\mu \mathrm{m}$ in this case) to maintain the slope quality for the two SMS-based edge filters with opposite spectral responses.

For the purpose of experimental verification, the two SMS-based edge filters were fabricated using a precision Fujikura CT-07 cleaver and a Sumitomo type-36 three-axis fusion splicer. For each SMS structure the process is the same. First, the input SMF and the input end of the MMF are cleaved and spliced together. To deliberately introduce an offset (and thus misalignment) in this splice, an attenuation splicing mode, available as an option on the fusion splicer, is used. This splicing mode allows for the creation of a fiber splice with a preset optical power loss. Given a preset power loss, the fusion splicer will automatically perform the splice with an appropriate axial offset.

The cleaver is again used to precisely cleave the un-terminated end of the MMF fiber so that its length is set to the desired value. Finally, the output end of MMF section is spliced to the cleaved end of the output SMF, again with the attenuation splicing option. The desired power loss is set so that it corresponds to an offset of $3.3 \mu \mathrm{m}$.

The transmission loss response of each fabricated filter is measured using a tunable laser and an optical spectrum analyzer (OSA). The measured results are shown in Fig. 7 for the 


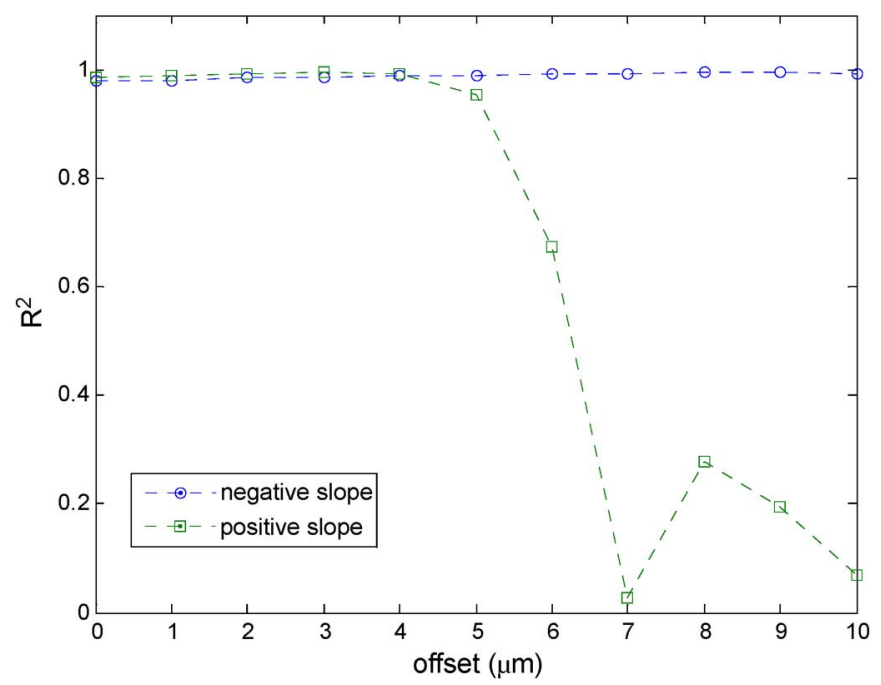

Fig. 6. Correlation coefficient of the spectral response for different offsets.

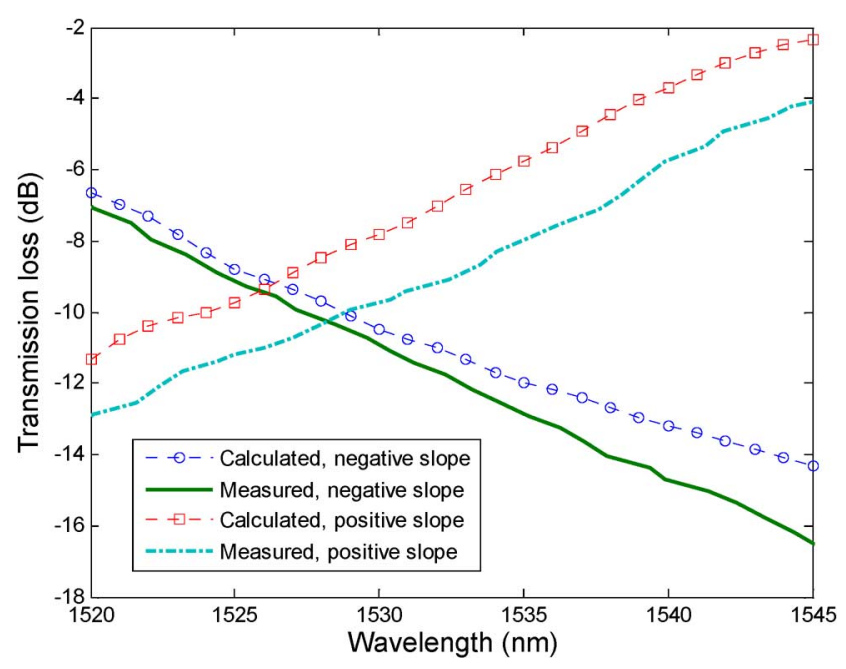

Fig. 7. Measured and calculated transmission loss with misalignment of the SMS-based edge filters.

negative and the positive slope SMS-based edge filters. The calculation of transmission loss using (4) is also shown in Fig. 7. The calculated and measured results show a good agreement. The discrepancy between the calculated and measured results is most likely a result of: 1) residual splicing insertion losses and 2) errors in the exact length of the MMF section. MMF section length errors, which arise during fabrication of the SMS structure, shift the peak wavelength of the bandpass filter response, which in turn alters the measured transmission loss values over a fixed wavelength range.

Experimentally the two edge filters with their deliberate misalignment, while they do possess a higher insertion loss, demonstrate response slopes with an acceptable discrimination ranges of 9.47 and $8.89 \mathrm{~dB}$ for the negative and positive slopes, respectively. The edge filters are therefore still suitable for use as edge filters. This result verifies our assertion that as long as the misalignment between SMF and MMF cores is less than an offset misalignment limit which is equal to the SMF core radius then there is no significant effect on the slope quality.

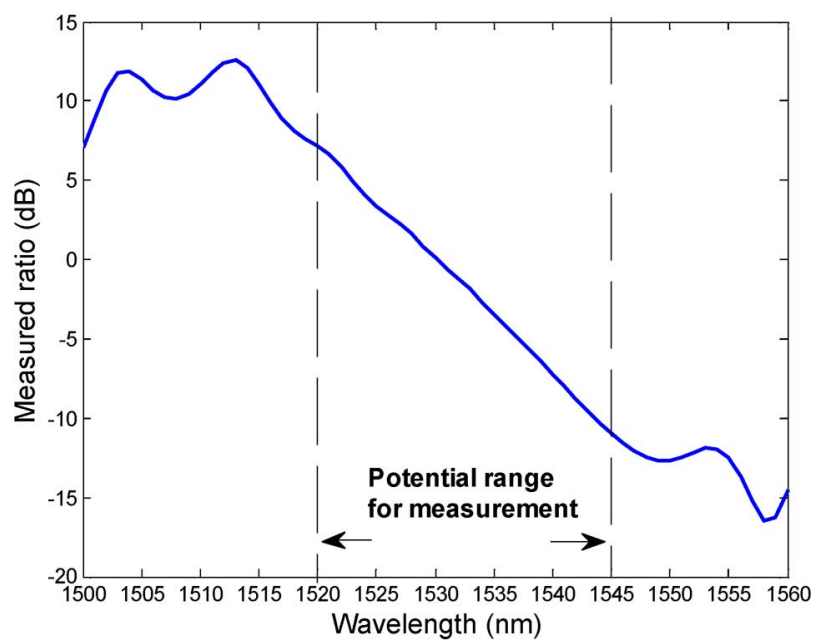

Fig. 8. Measured ratio.

To provide confirmation a misaligned SMS-based edge filter will work as long as the lateral misalignment in an SMS structure is less than the limit proposed, the edge filter described above with a $3.3 \mu \mathrm{m}$ lateral misalignment was employed in a functioning wavelength measurement system, based on the scheme described in Fig. 1(a). The input signal is split into two equal intensity signals using a $3 \mathrm{~dB}$ fiber splitter. One of the signals passes through the negative slope SMS-based edge filter and the other passes through the positive slope SMS-based edge filter. A dual channel power meter is placed at the ends of both arms. Fig. 8 shows the measured ratio of the optical power. The measured ratio between 1520 and $1545 \mathrm{~nm}$ has a linear slope with a discrimination range of $18.14 \mathrm{~dB}$ from 7.17 to $-10.97 \mathrm{~dB}$, which is suitable for wavelength measurement.

It should be noted that for the fabrication of an SMS, it is preferable to use a fusion splicing machine with the capability of three-axis adjustment rather than a single-axis (also called a fixed V-groove) fusion splicer. Lateral misalignment arises in an SMS structure for two reasons. First, there is the lateral misalignment introduced by the fusion splicer itself, and second, there is the misalignment that results from the limited manufacturing tolerance of core-cladding concentricity of the fibers used. Using a fusion splicer with three-axis adjustment can negate the effect of the limited core-cladding concentricity of the fibers. This means that the only significant source of misalignment is the inherent alignment accuracy of the fusion splicer itself. Typical three-axis adjustment splicers can maintain inherent misalignment to less than $0.5 \mu \mathrm{m}$ in the case of identical SMF or MMF splicing and thus can allow the fabrication of SMS structures with repeatably low lateral misalignment. The use of a single-axis fusion splicer is less advisable for SMS fabrication as it is not possible to overcome misalignment due to limited core concentricity and furthermore a single-axis splicer typically has an inherent misalignment that is higher than a three-axis adjustment splice machine.

\section{CONCLUSION}

The effect of misalignment on the spectral response of an SMS-based edge filter has been investigated. An MPA with a 
calculated set of guided modes using FDM is employed to analyze the misalignment effect. It is shown that the performance of the SMS based edge filter degrades when the lateral misalignment is larger than a misalignment limit which is equal to the core radius of the SMF used.The measured transmission loss responses show a good agreement with the numerical results. The SMS-based edge filter used in the experiment is found to be suitable for use in a wavelength measurement system. Overall, it is shown that an SMS structure fabricated using a fusion splicer with three-axis adjustment has the advantage of a useful fabrication tolerance, within which the lateral misalignment has no significant effect on the slope of edge filter.

\section{REFERENCES}

[1] Q. Wang and G. Farrell, "All-fiber multimode-interference-based refractometer sensor: Proposal and design," Opt. Lett., vol. 31, no. 3, pp. 317-319, 2006.

[2] W. S. Mohammed, P. W. E. Smith, and X. Gu, "All-fiber multimode interference bandpass filter," Opt. Lett., vol. 31, no. 17, pp. 2547-2549, 2006.

[3] Q. Wang and G. Farrell, "Multimode-fiber-based edge filter for optical wavelength measurement application and its design," Microw. Opt. Technol. Lett., vol. 48, no. 5, pp. 900-902, 2006.

[4] A. M. Hatta, G. Farrell, Q. Wang, G. Rajan, P. Wang, and Y. Semenova, "Ratiometric wavelength monitor based on singlemode-multimode-singlemode fiber structure," Microw. Opt. Technol. Lett., to be published.

[5] Q. Wang, G. Farrell, and W. Yan, "Investigation on singlemode-multimode-singlemode fiber structure," J. Lightw. Technol., vol. 26, no. 5, pp. 512-519, May 2008.

[6] H. Li, M. Brio, L. Li, A. Schülzgen, N. Peyghambarian, and J. V. Moloney, "Multimode interference in circular step-index fibers studied with the mode expansion approach," J. Opt. Soc. Amer. B, Opt. Phys., vol. 24, no. 10, pp. 2707-2720, 2007.

[7] K. Kawano and T. Kitoh, Introduction to Optical Waveguide Analysis: Solving the Maxwell Equations and the Schrodinger Equation. Hoboken, NJ: Wiley, 2001, pp. 117-160.

[8] S. M. Melle, K. Liu, and R. M. Measures, "Practical fiber-optic Bragg grating strain gauge system," Appl. Opt., vol. 32, no. 19, pp. 3601-3609, 1993.

[9] L. B. Soldano and E. C. M. Pennings, "Optical multi-mode interference devices based on self-imaging: Principles and applications," J. Ligthw. Technol., vol. 13, no. 4, pp. 615-627, Apr. 1995.

Agus Muhamad Hatta (S'09) received his B.Eng. degree in engineering physics and M.Sc. degree in optoelectronics-physics from Institut Teknologi Sepuluh Nopember (ITS) Surabaya, Indonesia, in 2002 and 2005 respectively.

$\mathrm{He}$ is currently on leave from the Engineering Physics Department, Institut Teknologi Sepuluh Nopember (ITS) Surabaya, Indonesia as a junior lecturer. Since 2006 he has been working toward his Ph.D. degree at Applied Optoelectronics Centre, Dublin Institute of Technology, Ireland. His research interest includes modeling and application of fiber optics and integrated optics for fast wavelength measurement and optical sensing.

Gerald Farrell graduated with an honours degree in electronic engineering from University College Dublin in 1979. He has a Ph.D. (1994) from Trinity College Dublin for research in all-optical synchronization using self-pulsating laser diodes that resulted in the development and patenting of a new method of all-optical frequency changing.

He spent a number of years as a communications systems design engineer before joining the DIT. He is the Head of the School of Electronic and Communications Engineering at the Dublin Institute of Technology (DIT). For the last 12 years Dr. Farrell has been the Director of the Applied Optoelectronics Centre at the DIT and also is the group leader for the Photonics Research group at the DIT. His research interests are in fast wavelength measurement for optical sensing; the modeling and analysis of FBG interrogation schemes and the modeling and application of fiber bend loss. He has authored or coauthored over 95 articles in journals and conferences and is a referee for a number of Optical and Photonic journals.

He is a principle investigator on several funded projects, some with international partners.

Pengfei Wang has been working toward his Ph.D. degree at the Applied Optoelectronics Centre Dublin Institute of Technology, Dublin, Ireland, since 2005.

His research interests include optoelectronics devices and the modeling and experimental investigations of bend loss in waveguides and fibers.

Ginu Rajan received his B.Sc. degree in physics from University of Kerala and M.Sc. degree in applied physics from Mahatma Gandhi University, Kerala, India, in 2000 and 2002, respectively. Since 2005 he has been working toward his Doctoral degree at Applied Optoelectronics Centre, Dublin Institute of Technology, Ireland.

He worked as a Research Trainee at Indian Institute of Astrophysics during the period 2003-2005. His research interest includes optical fiber sensors and its applications, fiber edge filters, FBG interrogation systems, and fast wavelength measurement techniques.

Yuliya Semenova graduated with a B.Sc. degree in electronic engineering from Lviv Polytechnic National University, Ukraine in 1992. She subsequently undertook research leading to a $\mathrm{Ph} . \mathrm{D}$. degree in physics of molecular and liquid crystals in 1999.

Between 1997 and 2001 she worked as a researcher at the faculty of Electrophysics, Lviv Polytechnic National University, Ukraine. Since 2001 she is a researcher and lecturer at the School of Electronic and Communications Engineering, Dublin Institute of Technology. Her research interests include physics of liquid crystals, their applications in photonics and fiber optic sensing. She has authored or coauthored over 40 articles in journals and conferences. 